\title{
Cooperative Catalysis for Asymmetric Decarboxylative Cyanation
}

Metal-Catalyzed

Asymmetric

Synthesis and

Stereoselective

Reactions

\section{Key words}

iridium catalysis

copper catalysis

photoredox

catalysis

decarboxylative

cyanation

hydroxyphthalimide

esters

alkyl nitriles

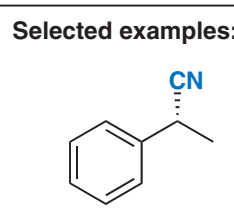

$81 \%$ yield, $89 \%$ ee<smiles>CC(C#N)c1ccc(P)cc1</smiles>

$38 \%$ yield, $94 \%$ ee<smiles>CCC(C#N)c1csc2ccccc12</smiles>

$67 \%$ yield, $88 \%$ ee Application:<smiles>CCOC(=O)C[C@H](C#N)c1ccccc1</smiles>

$90 \%$ ee<smiles>CC(C#N)c1ccc(Br)cc1</smiles>

$98 \%$ yield, $87 \%$ ee<smiles>CC(C#N)c1cccc2ccccc12</smiles>

$82 \%$ yield, $93 \%$ ee<smiles>CC(C#N)c1ccc(-c2ccccc2)c(F)c1</smiles>

$92 \%$ yield, $92 \%$ ee

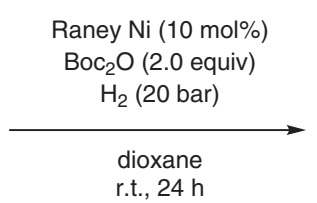<smiles>COc1ccc(C(C)C#N)cc1</smiles>

$71 \%$ yield, $82 \%$ ee<smiles>N#CC(Cl)c1ccccc1</smiles>

$93 \%$ yield, $96 \%$ ee<smiles>CC(C#N)c1ccc2c(c1)[nH]c1ccc(Cl)cc12</smiles>

$59 \%$ yield, $85 \%$ ee

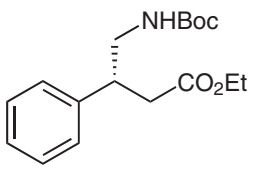

$92 \%$ yield, $89 \%$ ee<smiles>CC(C#N)c1ccc(Br)cc1</smiles>

$77 \%$ yield, $90 \%$ ee<smiles>CCOC(=O)C[C@H](C#N)c1ccccc1</smiles>

$88 \%$ yield, $90 \%$ ee<smiles>CC(C#N)c1ccc2c(c1)CC(=O)c1ccccc1S2</smiles>

$79 \%$ yield, $88 \%$ ee

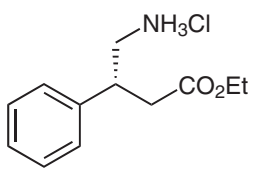

(R)-phenibut
Significance: Chiral alkyl nitriles are synthetically valuable compounds in organic synthesis. The authors have developed an asymmetric decarboxylative cyanation of $N$-hydroxyphthalimide esters by cooperative photoredox and copper catalysis.
Comment: This catalytic decarboxylative cyanation provides enantioenriched alkyl nitriles in good yields with high enantioselectivities. The reaction can be applied in the synthesis of a key intermediate for the chiral antidepressant molecule $(R)$ phenibut. 